
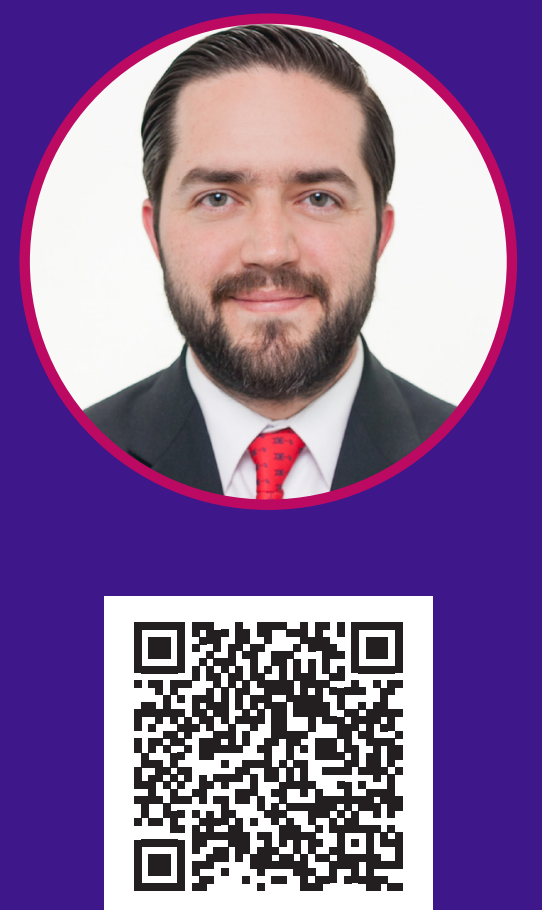

Videopresentación

\title{
La deuda del cine con la literatura
}

\author{
Cinema's debt to literature
}

Lic. Gustavo Echevarría Navarro

ITESM

gustavo.en@gmail.com

Recibido: 13 de agosto de 2020.

Aceptado: 13 de septiembre de 2020.
Received: August 13th 2020.

Accepted: September 13th 2020.

\section{(c) $(1) \ominus$ Esta obra está bajo una licencia internacional Creative EY NC ND Commons Atribución-NoComercial-SinDerivadas 4.0.}

DOI: https://doi.org/10.21555/rpc.voi2.2346

Cómo citar: Echevarría Navarro, G. . (2020). La deuda del cine con la literatura. RPC, (2), 129-134. https://doi. org/10.21555/rpc.v0i2.2346

Revista Panamericana de Comunicación, Año 2, N. 2, julio-diciembre 2020, pp. $129-134$.

\section{RESUMEN}

Es generalmente aceptado que el cine es un medio que ha sido influenciado, desde su origen y a lo largo de su historia, por otros medios artísticos. Son abundantes los estudios que tratan sobre instancias particulares de estas influencias en general, así como las consideraciones sobre obras cinematográficas específicas y su relación con otras producciones artísticas en el ámbito del teatro, la ópera o la danza. Al margen de un ejercicio de análisis narrativo concreto sobre alguna obra cinematográfica, en este ensayo nos aventuramos a proponer, utilizando la analogía y con fundamento antropológico, que, en última instancia, la primera génesis del cine como medio narrativo se encuentra en la literatura.

Palabras clave: cine, literatura, narrativa, ficción.

\section{ABSTRACT}

It is generally accepted that cinema has been influenced, from its origin and throughout its history, by other artistic media. There are abundant studies dealing with particular instances of these influences in general, as well as many considerations on specific cinematographic works and their relationship with other artistic productions in the field of theater, opera or dance. This essay focuses not on a narrative analysis of a specific film, instead we venture to propose, on the basis of analogy and anthropological grounds, that, ultimately, the first genesis of cinema as a narrative medium is found in literature.

Palabras clave: cinema, literatura, narrative, fiction. 


\section{PRIMER ACTO (A MODO DE INTRODUCCIÓN)}

El cine ${ }^{1}$ ¿le debe algo a la literatura? La pregunta no es tan solo retórica. Recientemente han surgido movimientos civiles que exigen resarcir daños históricos y sociales de una manera desconcertante, tanto por el modo de los reclamos como por el contenido de las denuncias. Estatuas han caído y personajes otrora llamados "ilustres" han sido denostados. Este contexto, marcado por manifestaciones tanto violentas como pacíficas, se nos presenta como una buena oportunidad para reflexionar sobre otras relaciones de poder, sobre el origen y las analogías existentes entre conductas socialmente aceptadas, o rechazadas, y algunas manifestaciones culturales que hemos llegado a aceptar como propias de nuestro momento histórico.

En Occidente nos gusta medir las cosas. Quien es capaz de presentar alguna métrica o relación proporcional es digno de recibir el curioso título de "experto". La vida cotidiana está plagada de preguntas que nos permiten medir y, eso esperamos, controlar nuestro entorno: ¿Cuánto cuesta? ¿De qué tamaño es o cuánto pesa? ¿Cuál es el tiempo de entrega?

En las industrias culturales procuramos conservar otros criterios de validación, quizá con la tenue esperanza de que las métricas "cualitativas" sigan justificando las partidas presupuestales públicas y los patrocinios privados. Incluso al margen de las modificaciones estructurales generadas por gobiernos populistas, o quizá con esa motivación, se sigue comparando -midiendo- a los distintos sectores culturales con la intención de determinar cuál industria es más importante, particularmente: ¿la del cine o la de la literatura? Más de uno se ha atrevido a buscar la cantidad de dinero que significa cada uno de estos mercados para dar una respuesta, pero una métrica monetaria es frecuentemente rechazada por los agentes protagonistas de estas artes. Por tratarse de fenómenos culturales 0 artísticos se suele acudir a calificaciones alternativas como el valor cul-

1 Para efectos prácticos, en el presente consideramos el término "cine" en modo genérico y amplio. De esta manera las reflexiones propuestas serían aplicables a cualquier producto de narrativa audiovisual de ficción, incluyendo las series televisivas o difundidas por plataformas de streaming, las web series, videojuegos, fenómenos transmedia, etc. tural, la expresión individual o social, la representación, inclusión o riqueza semiótica. Como se quiera medir, la comparación generalmente comprende al cine como el deudor en esta relación. ¿Por qué la literatura sería la parte acreedora? ¿Cuál sería esta deuda? ¿Cuál es el monto?

Para entender la pregunta inicial podemos plantear distintas perspectivas. La temporal nos ayuda muy poco, pues tenemos la mala suerte de que los hermanos Lumiere proyectaran sus diez películas hace unos pocos años, apenas más de cien, mientras que conocemos las aventuras del querido Gilgamesh desde hace más de cuatro milenios. La literatura es muy anterior al cine y esto a nadie sorprende; en este sentido el arte de la expresión verbal (R.A.E., 2017) se nos muestra como una actividad propiamente humana, pues tuvimos la buena suerte de que la literatura hiciera su maravillosa aparición desde muy pronto en nuestra historia.

Cuando los seres humanos pudimos, por fin, concentrarnos en educar a los críos y dejamos de estar a merced del clima para alimentarnos, o pendientes de la aparición de un hambriento depredador para trasladarnos, esto es, cuando finalmente el ser humano alcanzó el grado de desarrollo comunitario necesario para convertirse en una especie sedentaria, aparecieron los relatos taciturnos con el afán de explicar de dónde venimos, por qué somos los que somos y qué es lo que nos identifica como un grupo (Gottschall, 2012). No es un accidente que aquí identifiquemos los términos literatura y ficción: las historias que hemos compartido de generación en generación son el elemento más determinante en cuanto a la conformación de la especie humana como un grupo distinto al de los demás seres vivos.

Volviendo a la pregunta que motiva este ensayo, para saber si el cine tiene alguna deuda con la literatura tampoco nos sirve considerar los medios y las herramientas que utilizan. Con esto nos referimos a aquellos inventos que han permitido la conservación y transmisión de estas artes -los caracteres marcados en una superficie para la literatura, la película de celuloide para el cine-, que son accidentales y han surgido en contextos, momentos y circunstancias muy diferentes.

Por ejemplo, la literatura se empezó a plasmar en medios más duraderos que el habla, quizá, como remedio a la mala memoria de los adolescentes distraí- 
dos que no ponían toda su atención mientras los mayores narraban alrededor del fuego las historias de los antepasados. ¿Qué culpa tiene el pobre muchacho si esa chica de cabello alborotado, sentada al otro lado de la fogata, le había robado ya la imaginación?

El cine, en cambio, pudo existir porque al simpático Christiaan Huygens se le ocurrió fabricarse la lanterna mágica -nombre latino más simpático que el de su inventor, no cabe duda- por aquellos años del siglo XVII (Wikipedia contributors, Magic lantern). Escribir en una tablilla las historias que nos cuenta el líder de la tribu es, sin duda, tarea mucho más seria y loable que proyectar en la pared a la Muerte arrancándose la cabeza.

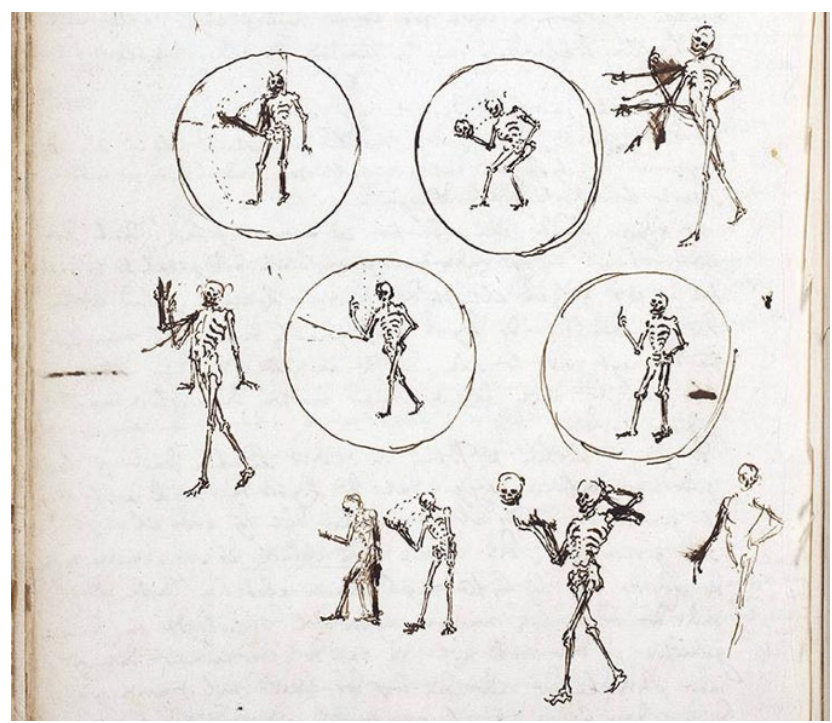

Figura 1. Caricatura de la muerte arrancándose la cabeza, realizada por Huygens para ser proyectada utilizando la linterna mágica.

No decimos que las herramientas carezcan de importancia, más bien al contrario pues el surgimiento de una nueva tecnología es, desde nuestro punto de vista, el único motivo por el que podemos disfrutar de las maravillosas historias proyectadas sobre una pantaIla tan grande como un edificio o tan pequeña como el teléfono que cargamos en el bolsillo. Pero nuestro argumento va más allá: si el cine está en deuda, en alguna clase de deuda, con la literatura no es por cuestiones temporales o cronológicas, tampoco por asuntos técnicos o cuestiones instrumentales.

\section{SEGUNDO ACTO (A MODO DE ARGUMENTACIÓN)}

Como hemos dicho, la labor artística cuyo fruto resultó en el ordenamiento y fijación de las historias más importantes para el grupo humano, esas narraciones que alimentaron tanto el tranquilo sueño de los ancianos como las intensas pesadillas de los más pequeños, configuraron, en unas pocas generaciones, la información básica y fundamental que permitía distinguir a "los nuestros" de "aquellos". Las dinámicas sociales entre un grupo y otro fueron evolucionando con base en la información socialmente relevante (Boyd, 2009) contenida en esas ficciones. Por esto es que la narrativa, en cualquiera de sus formas, es una actividad especialmente importante para la especie humana.

Mientras no tengamos la máquina del tiempo que nos permita viajar al pasado, nos faltará certeza sobre cuál fue el momento preciso en que el primer grupo de homo sapiens dijo por primera vez "nosotros" para distinguirse del pequeño núcleo que río abajo pretendía controlar un mayor territorio o hacerse de la mayor cantidad de recursos. De lo que sí estamos seguros es que ese momento no pudo ser muy distante a la primera noche en que una historia sirvió para educar a los vástagos sobre los riesgos de seguir con sus juegos más allá de donde las mujeres podían espiarlos. Si las historias nos enseñaron a funcionar como grupo y así, como grupo, es que el ser humano ha logrado desarrollarse y superar las limitaciones impuestas por el entorno, concluimos con Volpi (2011) que el arte no sólo es una prueba de nuestra humanidad: somos humanos gracias al arte.

El primer grupo humano descubrió muy pronto que la supervivencia es más asequible en la medida en que las tareas son distribuidas en función de las cualidades y aptitudes que más naturalmente corresponden a cada individuo: el fuerte y rudo cazador habrá preferido que unas manos más delicadas que las suyas arrancaran los frutos de las ramas más altas de aquel durazno. Esta primitiva especialización tuvo un producto inesperado, pero gratamente recibido, en el tiempo disponible para el ocio.

Esta especie, que ya no sólo reaccionaba al entorno sino que empezaba a transformarlo en favor 
de sus propias necesidades, pudo dar rienda suelta a la imaginación. Los relatos que se compartían en las reuniones nocturnas fueron tomando formas cada vez más complejas: el narrador utilizó distintas entonaciones y estructuras, se detuvo un poco más en la descripción de algún paisaje y quizá se divirtió a sí mismo introduciendo personajes nuevos a la historia que solo él había escuchado de su abuelo hacía unos años. De esta manera empezaron a surgir, como categorías de una expresión artística determinada -la literatura expresada oralmente- los diferentes géneros o modos de hacer una re-presentación. Salvo uno que otro coscorrón que quizá se llevó el más joven de los narradores al tergiversar de más una de las historias de los mayores, estos primeros experimentos fueron recibidos por nuestros primitivos ancestros con buenos ojos.

En resumen, la ficción literaria debe ser considerada una adaptación evolutiva que, animada por la dinámica cooperativa propia de un grupo social, nos permite evaluar nuestra conducta en situaciones futuras, conservar la memoria individual y colectiva, comprender y ordenar los hechos a través de secuencias narrativas y, en última instancia, introducirnos en las vidas de los otros, anticipar sus reacciones y descifrar su voluntad y sus deseos. (Muñoz García, 2007)

De esta manera, como un experimento que quizá surgió primero de un accidente y después se fue perfeccionando con base en alguna astuta curiosidad, surgieron los primeros géneros literarios.

"Cuéntame esa historia que me hace reír" habrán sido deliciosas palabras para el primer narrador cómico; la solicitud "mejor esa otra de los mejores cazadores de nuestra tribu" seguramente hinchó el pecho del creador de la épica. No hay lugar en estas páginas para detenernos con clasificaciones, subdivisiones y consideraciones profundas sobre los distintos géneros que la delicada señora Literatura ha tenido a bien parir. Baste por ahora presentar una idea bastante rala: en cuanto la narrativa y la ficción tuvieron un lugar reconocido entre las actividades humanas, la literatura comenzó a experimentar transformaciones y evoluciones sucesivas que dieron pie, en primera instancia, a los tres géneros madre. La épica, la lírica y el drama alimentaron el espíritu de nuestros primeros antepasados, de tal modo que, durante algunos milenios, fueron el campo fértil donde se cosecharon los más grandes avances sociales, políticos y, sobre todo, artísticos.

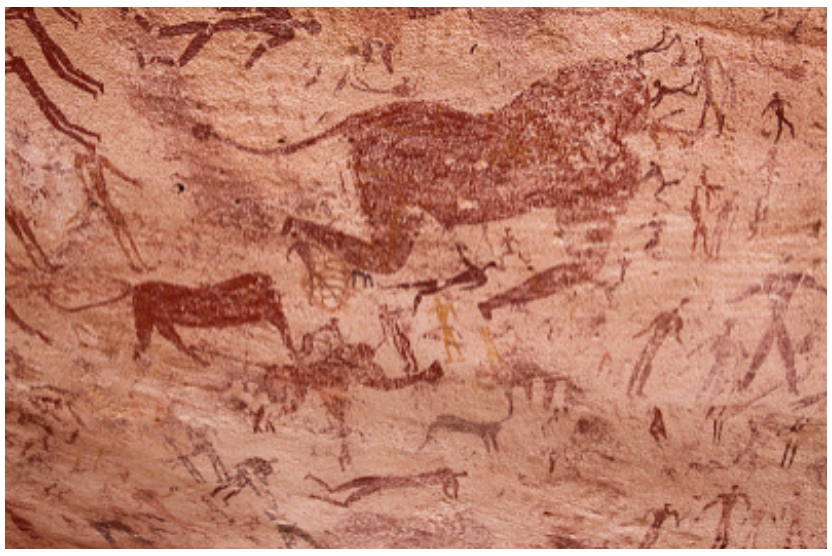

Figura 2. Seres humanos bailando, flotando y nadando alrededor de unas bestias. Pintura rupestre fijada sobre los muros de la Cueva de las Bestias en Wadi Sura, Egipto.

\section{TERCER ACTO (A MODO DE CONCLUSIÓN)}

Combinaciones, mezclas, accidentes y uno que otro intento demasiado arriesgado produjeron un sinfín de ficciones contadas una y otra vez. Distintos personajes y tramas encontraron un lugar en la memoria y la imaginación de quienes ya no escuchaban sentados alrededor de una fogata, sino sentados en sus casas, construidas con materiales más resistentes dentro de una ciudad amurallada. En unos cuantos siglos los autores empezaron a batallar por encontrar nuevas historias. ¡Cuánta lástima nos da el primer escritor al que no se le ocurrió nada nuevo porque ya todo había sido escrito por los demás! Los héroes ya no tienen más doncellas a quienes rescatar ni hay más reyes que puedan ofrecer otras recompensas. Todas las historias de amor han sido ya contadas, olvidadas y vueltas a recordar.

Cambian los personajes, los lugares, el tiempo y algún otro detalle pero desde hace mucho ya no hay nada nuevo bajo el sol que le da luz y calor a la ficción. Quizá por este motivo es que la novela en el siglo veinte recibió una bocanada de aire menos mohoso -que 
no precisamente fresco- de la mano de Proust, Joyce y Kafka, sin embargo ellos no hicieron más que rebuscar y volver a rascar en el fondo del cajón de las herramientas olvidadas de los escritores: sus personajes son más profundos porque se vuelven sobre ellos mismos, rompen con la linealidad del tiempo narrativo, prestan más atención a las acciones interiores de los protagonistas y experimentan colocando al narrador en lugares y perspectivas inesperadas. En otras palabras, sus obras resultan interesantes y relevantes porque cuentan las mismas cosas de manera distinta.

Todas las historias han sido contadas. Esta idea podría espantar o deprimir a más de alguno, pero que no cunda el pánico: todas las caras son muy parecidas entre sí, tienen dos ojos con sus cejas y pestañas, una nariz, una boca... pero de alguna manera seguimos disfrutando la figura distinta de los diferentes rostros $y$, de vez en cuando, hasta nos enamoramos de alguno. (Gottschall, 2012)

Ahora bien, sin considerar detenidamente lo que constituye un lenguaje o una lengua, pensamos en el cine como en el muchacho adolescente que fue invitado - llegó obligado y con la firme determinación de no pasarla bien- a la reunión familiar de las expresiones artísticas. En el rincón de allá las solteronas Pintura y Escultura, bien entradas en años pero sin una sola arruga a la vista, se ríen al recordar cuando su tía segunda, la gorda Ópera, arruinó la reunión familiar de diciembre al obsequiar a cada quien una fotografía de ella misma en sus vacaciones por la Toscana. Sentados en el estudio, los tíos Teatro y Canto miran, arrullados por el whisky y todavía con un carajillo en la mano, el partido de fútbol. En la cocina no paran las hermanas Poesía y Música, pues el pastel se desinfló y están preparando un postre improvisado para servir el café. La abuela, la querida Nana Lite como le llaman todos cariñosamente, se pone de pie al ver entrar a Cine que con los audífonos puestos -esperaba poder llegar hasta el jardín sin tener que detenerse a saludar a nadie- y después de dos tronados besotes en las mejillas le recuerda lo bien que se ve y cómo, de seguro mijito, todas las niñas de la escuela le piden que las lleve al baile o a esa cosa... ¿el antro?

En la casa de la abuela caben todos y siempre está llena de gente peculiar. Por eso es que a nuestro atormentado adolescente no le gusta mucho estar ahí: sabe que lo quieren, pero todos le preguntan por sus planes a futuro... quieren saber qué está haciendo para, desde ahora o en el futuro, ser alguien, un hombre de bien... ¿Acaso no se dan cuenta? ¡Ni él mismo lo sabe todavía! No sabe si prefiere proyectarse en pantallas gigantescas o si se siente más cómodo en los aparatos que caben en la palma de la mano. ¿Es mejor que se le pueda ver en cualquier momento 0 , por el contrario, se debería acudir a un lugar expresamente diseñado, y repleto de extraños, para observarlo en los horarios determinados por los estudios de mercado? Al cine le gustaría tener claros sus propios planes, mientras tanto le basta con copiar, a escondidas, lo que desde muy chiquito le ha contado Nana Lite que ella hacía en su infancia y juventud. Por ahora le basta seguir contando historias $y$, a veces más, a veces menos, experimentar con uno u otro modo de hacer eso mismo.

Si resulta que lo que decimos tiene algún correlato con la realidad y todos los géneros, así como todas las historias, ya están contenidos en la literatura de una o de otra forma, entonces el invitado que llegó tarde a la fiesta, el cine, no tiene otro remedio, por ahora, que dejarse llevar por lo que está sucediendo. Los géneros cinematográficos han surgido como adaptaciones e interpretaciones de los géneros literarios que son traídos a este nuevo medio, el film.

En todo caso, así es como el cine tiene una deuda con la literatura. Es el adolescente de la familia la familia de las artes- que, si no fuera porque su abuela engendró, no existiría. El cine experimenta y, mientras encuentra su propio camino, está parcialmente satisfecho con los resultados que va obteniendo. A veces piensa que es muy original y creativo, se convence a sí mismo de estar haciendo cosas que a nadie más se le han ocurrido, para desencantarse pronto al volver a casa de la abuela y encontrar en el rincón un empolvado recuerdo de eso mismo que él pensaba haber creado ex nihilo. El cine le debe a la literatura lo que todo adolescente le debe a su abuela: absolutamente todo y, desde su punto de vista, absolutamente nada. I 


\section{MISCELÁNEA}

\section{REFERENCIAS}

» Boyd, B. (2009). On the Origin of Stories: Evolution, Cognition, and Fiction. Harvard University Press.

» Escalonilla, A. S. (2011). Fantasía de aventuras: La exploración de Universos fantásticos en literatura y cine. Communication \& Society, 22(2), pgs. 109-137.

» Gottschall, J. (2012). The Storytelling Animal: How Stories Make Us Human [Kindle iOS version]. Retrieved from www.amazon. com

» Muñoz García, J. J. (2007). El cine como experiencia antropológica y ética. Revista de Comunicación, 6, pgs. 17-35.

» Pirandello, L. (2015). Seis Personajes en busca de autor. [Kindle iOS version]. Retrieved from www.amazon.com

» Real Academia Española. (Actualización 2017) Literatura. http:// dle.rae.es/?id=NR70JFI

»Volpi, J. (2011). Leer la mente: El cerebro y el arte de la ficción [Kindle iOS version]. Retrieved from www.amazon.com

» Wikipedia contributors. (2018, October 15). History of film. In Wikipedia, The Free Encyclopedia. https://en.wikipedia.org/w/index. php?title=History_of_film\&oldid=864167710

» Wikipedia contributors. (2018, October 15). Magic lantern. In Wikipedia, The Free Encyclopedia. https://en.wikipedia.org/w/index. php?title=Magic_lantern\&oldid=864162019

» Zavala, L. (2016). De qué hablamos al decir «estética del cine». Desde El Sur. Revista de Ciencias Humanas y Sociales de La Universidad Científica Del Sur, 08(01), pgs. 85-100. https://doi. org/10.21142/des-0801-2016-85-100 